\title{
MORTALIDAD NEONATAL, ANÁLISIS DE REGISTROS DE VIGILANCIA E HISTORIAS CLINNICAS NEONATALES DEL AÑO 2011 EN HUÁNUCO Y UCAYALI, PERÚ
}

\author{
José Enrique Velásquez Hurtado 1,2,a,c, Lourdes Kusunoki Fuero ${ }^{1, a, b}$, Tania Gisella Paredes Quiliche ${ }^{1, a, c, d}$, \\ Raquel Hurtado La Rosa ${ }^{1,2, c, f}$, Ángel Martín Rosas Aguirre ${ }^{2, a, c}$, Walter Eduardo Vigo Valdez $z^{1, a, e}$
}

\begin{abstract}
RESUMEN
Objetivos. Estimar la tasa de mortalidad neonatal y describir las defunciones neonatales ocurridas en el 2011 en hospitales del Ministerio de Salud de las regiones Huánuco y Ucayali, Perú. Materiales y métodos. Estudio transversal realizado de septiembre a noviembre de 2012 en Huánuco y Ucayali. Se revisaron los registros de las defunciones neonatales ocurridas en el 2011 en municipalidades provinciales, direcciones regionales de salud y cuatro hospitales de referencia. Para el cálculo de las tasas de mortalidad se utilizaron las fuentes de información más confiables por región. La revisión de 185 historias clínicas en los hospitales permitió describir las causas básicas de las muertes neonatales. Resultados. En el 2011 se reportaron en Huánuco 10886 recién nacidos vivos y 158 muertes neonatales, con una tasa de 14,5 muertes por 1000 nacidos vivos. En Ucayali, se reportaron 11441 recién nacidos vivos y 138 muertes neonatales, con una tasa de 12,1 muertes por 1000 nacidos vivos. La mayoría de muertes neonatales hospitalarias ocurrieron en los primeros 7 días de vida (87\%), en neonatos prematuros $(73,9 \%)$ y con bajo peso al nacimiento $(67 \%)$. Las causas básicas más frecuentes en las muertes neonatales fueron: infección $(31,4 \%)$, malformación congénita $(22,2 \%)$ y prematuridad $(18,9 \%)$. Conclusiones. La tasa de mortalidad neonatal en las regiones estudiadas fue superior a la media nacional. Los resultados sugieren la necesidad de intervenciones efectivas e integrales durante el embarazo, parto y periodo posnatal temprano; siendo este último periodo el de mayor vulnerabilidad en el neonato.
\end{abstract}

Palabras clave: Recién nacido; Mortalidad neonatal; Causas de muerte; Salud materno-infantil; Prevención y control (fuente: BIREME).

\section{NEONATAL MORTALITY, ANALYSIS OF SURVEILLANCE REGISTERS, AND NEONATAL MEDICAL HISTORIES OF 2011 IN HUANUCO AND UCAYALI, PERU}

\begin{abstract}
Objectives. To estimate the rate of neonatal mortality and to describe neonatal deaths in 2011 in hospitals of the Ministry of Health at Huanuco and Ucayali (Peru). Materials and methods. Cross-sectional study from September to November 2012 in Huanuco and Ucayali. Records of neonatal deaths in 2011 were reviewed from provincial municipalities, regional health directorates and four referral hospitals. To calculate mortality rates, we used the most reliable information sources by region. Reviewing 185 medical records in hospitals allowed us to describe the root causes of neonatal deaths. Results. In 2011, 10,886 live births and 158 neonatal deaths were reported in Huanuco, with a rate of 14.5 deaths per 1000 live births. In Ucayali, 11,441 live births and 138 neonatal deaths were reported, with a rate of 12.1 deaths per 1000 live births. Most hospital neonatal deaths occurred in the first 7 days of life (87\%), in preterm infants $(73.9 \%)$ and with low birth weight $(67 \%)$. The most common underlying causes of neonatal deaths were infection $(31.4 \%)$, congenital malformation $(22.2 \%)$ and prematurity $(18.9 \%)$. Conclusions. Neonatal mortality rate in the studied regions was higher than the national average. The results suggest the need for effective and comprehensive interventions during pregnancy, childbirth and the early postnatal period; this last period is the most vulnerable in the neonate.
\end{abstract}

Key words: Infant, newborn; Neonatal mortality; Cause of death; Maternal and child health; Prevention and control (source: MeSH NLM).

\footnotetext{
Programa de Apoyo a la Reforma del Sector Salud (PARSALUD II). Ministerio de Salud. Lima, Perú.

Universidad Peruana Cayetano Heredia. Lima, Perú.

Médico cirujano; ${ }^{b}$ Pediatra; ' Magíster en Salud Pública; ${ }^{\mathrm{d}}$ Magíster en Bioestadística; ${ }^{\mathrm{e}}$ Magíster en Gerencia de Proyectos y Programas Sociales;

${ }^{\mathrm{f}}$ Doctorado en Salud Pública

Recibido: 07-01-14 Aprobado: 23-04-14
}

Citar como: Velásquez Hurtado JE, Kusunoki Fuero L, Paredes Quiliche TG, Hurtado La Rosa R, Rosas Aguirre AM, Vigo Valdez WE. Mortalidad neonatal, análisis de registros de vigilancia e historias clínicas neonatales del año 2011 en Huánuco Y Ucayali, Perú. Rev Peru Med Exp Salud Publica. 2014;31(2):228-36. 


\section{INTRODUCCIÓN}

Durante el año 2011, se estimó que alrededor de 135 millones de bebés nacieron vivos en el mundo, 3 millones de ellos fallecieron durante los primeros 28 días (1). Si bien se han presentado avances en la reducción de la mortalidad infantil durante la década anterior, podemos apreciar que la mortalidad neonatal constituye aproximadamente el $43 \%$ de la mortalidad de los niños menores de 5 años en general. Esto pone a la mortalidad neonatal como una prioridad política constante, aun luego de alcanzados los Objetivos de Desarrollo del Milenio relacionados con la mortalidad infantil en algunos lugares.

En los últimos años, el Perú ha mostrado importantes logros en materia de salud pública, particularmente relacionados con la salud de los niños menores de un año, lo que ha permitido que el país se aproxime al cumplimiento de las metas de los Objetivos de Desarrollo del Milenio, relativas a la reducción de la mortalidad infanti( ${ }^{(2)}$. Sin embargo, la mortalidad neonatal ha tenido una velocidad de reducción bastante menor, representando en la actualidad más de la mitad de las causas de muerte en el primer año de vida ${ }^{(3)}$.

Las muertes neonatales ocurren con mayor frecuencia en lugares con elevados niveles de pobreza, ruralidad, analfabetismo y limitado acceso a los servicios de salud $^{(4)}$. Algunos de los factores descritos que conducen a mortalidad neonatal son: la falta de un control adecuado del embarazo y parto; la dificultad para reconocer signos de alarma en recién nacidos para búsqueda oportuna de ayuda profesional, y un repertorio limitado de intervenciones para tratar trastornos neonatales tempranos como la asfixia en el parto y los problemas derivados del parto prematuro ${ }^{(5,6)}$.

A pesar de los esfuerzos nacionales y regionales en el control de la mortalidad neonatal, las realidades regionales y locales son heterogéneas. Por ello, la adecuada vigilancia de la tasa de mortalidad neonatal, el período de la muerte, así como de los factores causales podrían facilitar el fortalecimiento y seguimiento de las intervenciones preventivas y curativas que se implementan a nivel comunitario y en los servicios de salud, para mejorar el cuidado y atención de la madre y el recién nacido.

En el marco de la intervención de la Segunda Fase del Programa de Apoyo a la Reforma del Sector Salud (7), se realizó un estudio en las regiones Huánuco y Ucayali, que tuvo como objetivos estimar la tasa de mortalidad neonatal y describir las características de las defunciones neonatales ocurridas durante el año 2011 en hospitales del Ministerio de Salud de ambas regiones.

\section{MATERIALES Y MÉTODOS}

Estudio observacional transversal realizado en Huánuco y Ucayali. Se revisaron los registros disponibles de defunciones neonatales ocurridas durante el año 2011 en los registros civiles de las municipalidades provinciales de Huánuco, Amarilis, Leoncio Prado, Coronel Portillo y Yarinacocha; en las direcciones regionales de salud (DIRESA), y en los servicios de neonatología de los establecimientos de salud que reportan la mayor cantidad de nacimientos y defunciones neonatales: Hospital Regional Hermilio Valdizán (HRHV), Hospital de Tingo María (HTM) y Centro de Salud Carlos Showing (CSCS) en Huánuco; y Hospital Regional de Pucallpa (HRP) y Hospital Amazónico de Yarinacocha (HAY) en Ucayali.

La tasa de mortalidad neonatal en cada región fue calculada mediante la división del número de nacidos vivos fallecidos en los primeros 28 días de vida (numerador) entre el número total de nacidos vivos (denominador) por 1000. El numerador incluyó todas las defunciones registradas en los servicios de neonatología de los establecimientos de salud seleccionados, así como las defunciones registradas en establecimientos no seleccionados. La comparación de los datos de mortalidad neonatal entre las diferentes áreas encargadas de consolidar y sistematizar dicha información en la DIRESA (epidemiología, estadística e informática, salud sexual y reproductiva), así como de los registros civiles de las municipalidades; con los registros de los establecimientos de salud seleccionados se pudo identificar la fuente de información más confiable a nivel regional. Debido a que no hubo revisión de registros en establecimientos de salud no seleccionados, el número de defunciones en ellos fue estimado usando la fuente identificada como más confiable para la región. Posteriormente, se revisaron las historias clínicas disponibles de los neonatos fallecidos en el año 2011 en los establecimientos de salud seleccionados. Con la finalidad de caracterizar adecuadamente las defunciones, los datos fueron recolectados en una ficha de datos diseñada para el estudio que contenía las siguientes variables: edad materna, grado de instrucción de la madre, número de controles prenatales de la madre, antecedente clínico materno, lugar y tipo de parto, tiempo de vida del neonato fallecido, edad gestacional al nacimiento, peso al nacimiento, puntuación APGAR, motivo de ingreso a servicio de neonatología y causa de muerte.

La recolección de datos y el posterior análisis de cada de muerte neonatal fueron realizados por dos médicos pediatras con experiencia en el manejo de historias clínicas y de trabajo asistencial en servicios de neonatología. Los médicos expertos consideraron todos 
Tabla 1. Número de muertes neonatales registradas en servicios de salud seleccionados de Huánuco y Ucayali según fuente de información, Perú 2011

\begin{tabular}{|c|c|c|c|c|c|}
\hline \multirow{2}{*}{ Región } & \multirow{2}{*}{$\begin{array}{l}\text { Registro del } \\
\text { servicio de salud }\end{array}$} & \multicolumn{3}{|c|}{ Dirección General de Salud } & \multirow{2}{*}{$\begin{array}{l}\text { Registro civil } \\
\text { de municipios }\end{array}$} \\
\hline & & Epidemiología & Estadística & Salud Sexual y Reproductiva & \\
\hline \multicolumn{6}{|l|}{ Huánuco } \\
\hline HTM & 26 & 25 & 16 & NR & 21 \\
\hline HRHV & 52 & 48 & 48 & NR & 38 \\
\hline CSCS & 0 & 0 & 0 & NR & 0 \\
\hline \multicolumn{6}{|l|}{ Ucayali } \\
\hline HAY & 53 & 46 & 46 & 49 & 34 \\
\hline HRP & 70 & 49 & 49 & 68 & 31 \\
\hline
\end{tabular}

HTM: Hospital de Tingo María. HRHV: Hospital Regional Hermilo Valdizán. CSCS: Centro de Salud CArlos Showing. HAY: Hospital Amazónico de Yarinacocha. HRP: Hospital Regional de Pucallpa. NR: no registrado

los registros incluidos en las historias clínicas (registros médicos, de enfermería, resultados de exámenes auxiliares, etc.) para asignar en forma independiente una causa básica para cada muerte neonatal siguiendo el procedimiento estándar recomendado por la Organización Mundial de la Salud para completar el certificado médico de defunción ${ }^{(8)}$. Se consideró causa básica a aquella enfermedad que inició la cadena de acontecimientos patológicos que condujo directamente a la muerte. La causa básica para cada muerte neonatal fue seleccionada de un total de ocho posibles: prematuridad, infección, asfixia, insuficiencia respiratoria, insuficiencia cardiaca, malformación, otra, y no determinada. Cuando hubo discordancias en la causa básica de las muertes neonatales (menos del $10 \%$ de los casos), se buscó el consenso entre los médicos expertos. Los datos registrados en la ficha de datos fueron ingresados a una hoja de cálculo utilizando el programa MS Excel@, con dos digitadores independientes. El procesamiento y posterior análisis se hicieron en el paquete estadístico SPSS ${ }^{\circ}$ versión 18.0. Las características y causas de muerte de los neonatos fueron expresadas por región en frecuencias absolutas y relativas. Se utilizó la prueba de chi cuadrado para determinar asociaciones entre el momento del fallecimiento del neonato (menos de
24 h, de 1 a 7 días, más de 7 días de nacidos) y otras características importantes como: peso al nacimiento (menor de 1500 g, de 1500 a 2499 g, y 2500 gramos o más); edad gestacional (menos de 32 semanas, de 32 a 36 semanas, y 37 semanas o más), y causa básica de defunción. En todos los casos se consideró un $p<0,05$ como significativo.

\section{RESULTADOS}

Se encontraron diferencias de datos de mortalidad neonatal en el 2011 entre las áreas encargadas de consolidar y sistematizar la información en la DIRESA (epidemiología, estadística e informática, salud sexual y reproductiva). La base de datos de mortalidad perinatal de la Oficina de Epidemiología de la DIRESA Huánuco, y los informes de salud materno-perinatal del Área de Salud Sexual y Reproductiva de la DIRESA Ucayali fueron las fuentes de información más confiables en Huánuco y Ucayali respectivamente, los datos más cercanos a los reportados por los establecimientos de salud (Tabla 1). Por el contrario, la revisión de los registros civiles de las municipalidades evidenció un alto subregistro de la mortalidad neonatal (cercano al 40\%).

Tabla 2. Tasas de mortalidad neonatal en las regiones Huánuco y Ucayali, Perú 2011

\begin{tabular}{lccc}
\hline \multicolumn{1}{c}{ Región } & $\begin{array}{c}\text { Recién nacidos } \\
\text { vivos }\end{array}$ & $\begin{array}{c}\text { Muertes neonatales } \\
\text { reportadas }\end{array}$ & $\begin{array}{c}\text { Tasa mortalidad (por 1000 } \\
\text { nacidos vivos) }\end{array}$ \\
\hline Huánuco & & & 12,2 \\
$\quad$ Hospital de Tingo María & 2125 & 26 & 21,6 \\
Hospital Regional Hermilio Valdizán & 2409 & 52 & 0,0 \\
Centro de Salud Carlos Showing & 680 & 0 & 14,1 \\
Otros establecimientos de salud & 5672 & 80 & 14,5 \\
$\quad$ Total región & 10886 & 158 & 15,8 \\
Ucayali & & 53 & 20,7 \\
Hospital Amazónico de Yarinacocha & 3358 & 70 & 3,2 \\
Hospital Regional de Pucallpa & 3389 & 15 & 12,1 \\
\hline Otros establecimientos de salud & 4694 & 138 & \\
Total región & 11441 & & \\
\hline
\end{tabular}

\footnotetext{
${ }^{*}$ Establecimientos no seleccionados.
} 
Durante el 2011 se reportaron 10886 recién nacidos vivos y 158 muertes neonatales en Huánuco, estimándose una tasa de 14,5 muertes por 1000 nacidos vivos (Tabla 2 ). Cerca de la mitad de muertes $(49,4 \%)$ se reportaron en los hospitales HTM y HRHV, con tasas de mortalidad neonatal de 12,2 y 21,6 por 1000 nacidos vivos, respectivamente. Mientras, que la mitad restante de muertes $(50,6 \%)$ fueron registradas en el resto de establecimientos no seleccionados de la región. En Ucayali se reportaron 11441 recién nacidos vivos y 138 muertes neonatales, con una tasa de 12,1 muertes neonatales por 1000 nacidos vivos. La mayoría $(89,1 \%)$ de muertes se concentraron en los hospitales HAY y HRP, con tasas de mortalidad neonatal de 15,8 y 20,7 por 1000 nacidos vivos, respectivamente. Solo el $10,9 \%$ de las muertes fueron registradas en establecimientos no seleccionados.
Se revisaron 185 historias clínicas del total de 201 muertes neonatales registradas en los hospitales en el 2011: 26 en HTM; 41 en HRHV; 48 en HAY, y 70 en HRP. Nueve historias clínicas no fueron encontradas y siete tenían datos incompletos o incongruentes. La edad materna de los recién nacidos fallecidos fue $24 \pm 7,7$ años en Huánuco y $25,1 \pm 7,6$ años en Ucayali, con predominio del grupo de 18 a 25 años en ambas regiones (Tabla 3). En Huánuco, solo el $43,5 \%$ de las madres había alcanzado el nivel de instrucción secundaria, mientras que en Ucayali el porcentaje de madres con nivel secundario alcanzó el $62,7 \%$. En ambas regiones, solo la cuarta parte de madres habían cumplido seis o más controles prenatales, y alrededor del $10 \%$ del total de las madres no asistieron a control prenatal. Más del $90 \%$ de madres dieron a luz en un establecimiento de salud; siendo la proporción de parto por cesárea $20,9 \%$ en Huánuco y $32,4 \%$ en Ucayali.

Tabla 3. Antecedentes maternos y del parto de los neonatos fallecidos en hospitales de Huánuco y Ucayali, Perú 2011

\begin{tabular}{|c|c|c|c|c|c|c|}
\hline & \multicolumn{2}{|c|}{ Huánuco } & \multicolumn{2}{|c|}{ Ucayali } & \multicolumn{2}{|c|}{ Total } \\
\hline & $\mathrm{N}=67$ & $(\%)$ & $\mathrm{N}=118$ & $(\%)$ & $n=185$ & $(\%)$ \\
\hline \multicolumn{7}{|l|}{ Edad materna } \\
\hline Menor de 18 años & 11 & $(18,0)$ & 15 & $(17,4)$ & 26 & $(17,7)$ \\
\hline 18 a 25 años & 28 & $(45,9)$ & 36 & $(41,9)$ & 64 & $(43,5)$ \\
\hline 26 a 35 años & 15 & $(24,6)$ & 26 & $(30,2)$ & 41 & $(27,9)$ \\
\hline Mayor de 35 años & 7 & $(11,5)$ & 9 & $(10,5)$ & 16 & $(10,9)$ \\
\hline NE/NR & 6 & & 32 & & 38 & \\
\hline \multicolumn{7}{|l|}{ Educación materna } \\
\hline Primaria & 20 & $(43,5)$ & 25 & $(30,1)$ & 45 & $(34,9)$ \\
\hline Secundaria & 20 & $(43,5)$ & 52 & $(62,7)$ & 72 & $(55,8)$ \\
\hline Superior & 6 & $(13,0)$ & 6 & $(7,2)$ & 12 & $(9,4)$ \\
\hline NE/NR & 21 & & 35 & & 56 & \\
\hline \multicolumn{7}{|c|}{ Número de controles prenatales } \\
\hline Ninguno & 9 & $(17,3)$ & 4 & $(5,4)$ & 13 & $(10,3)$ \\
\hline 1 a 2 & 13 & $(25,0)$ & 21 & $(28,4)$ & 34 & $(27)$ \\
\hline 3 a 5 & 17 & $(32,7)$ & 30 & $(40,5)$ & 47 & $(37,3)$ \\
\hline 6 o más & 13 & $(25,0)$ & 19 & $(25,7)$ & 32 & $(25,4)$ \\
\hline NE/NR & 15 & & 44 & & 59 & \\
\hline \multicolumn{7}{|c|}{ Antecedentes clínicos maternos } \\
\hline Anemia & 0 & $(0)$ & 2 & $(4,3)$ & 2 & $(2,1)$ \\
\hline Patología placentaria & 4 & $(8,3)$ & 0 & $(0)$ & 4 & $(4,3)$ \\
\hline Infección urinaria & 14 & $(29,2)$ & 2 & $(4,3)$ & 16 & (17) \\
\hline Hipertensión arterial & 0 & $(0)$ & 1 & $(2,2)$ & 1 & $(1,1)$ \\
\hline Eclampsia & 3 & $(6,3)$ & 1 & $(2,2)$ & 4 & $(4,3)$ \\
\hline Diabetes & 1 & $(2,1)$ & 0 & (0) & 1 & $(1,1)$ \\
\hline Sífilis & 1 & $(2,1)$ & 0 & (0) & 1 & $(1,1)$ \\
\hline VIH & 2 & $(4,2)$ & 1 & $(2,2)$ & 3 & $(3,2)$ \\
\hline Ninguna de las anteriores & 23 & $(47,9)$ & 39 & $(84,8)$ & 62 & (66) \\
\hline NE/NR & 19 & & 72 & & 91 & \\
\hline \multicolumn{7}{|l|}{ Lugar de parto } \\
\hline Institucional & 65 & $(97,0)$ & 95 & $(89,6)$ & 160 & $(92,5)$ \\
\hline Domiciliario & 2 & $(3,0)$ & 11 & $(10,4)$ & 13 & $(7,5)$ \\
\hline NE/NR & 0 & & 12 & & 12 & \\
\hline \multicolumn{7}{|l|}{ Tipo de parto } \\
\hline Vaginal & 53 & $(79,1)$ & 78 & $(67,6)$ & 131 & $(74,0)$ \\
\hline Cesárea & 14 & $(20,9)$ & 32 & $(32,4)$ & 46 & $(26,0)$ \\
\hline NE/NR & 0 & & 8 & & 8 & \\
\hline
\end{tabular}

* NE/NR $=$ No especificado, no registrado 
Más de las dos terceras partes de los neonatos fallecidos tuvieron bajo peso (menos de $2500 \mathrm{~g}$ ) o fueron prematuros al nacimiento (menos de 37 semanas de edad gestacional) (tabla 4). Del total de 122 neonatos con bajo peso al nacer, 111 (90,9\%) fueron, además, prematuros. En Huánuco, 59 (88,1\%) muertes neonatales ocurrieron en la primera semana de vida, 19 $(28,4 \%)$ dentro de las primeras 24 h. En Ucayali, 102 $(86,5 \%)$ muertes neonatales ocurrieron en la primera semana, $48(40,7 \%)$ en las primeras $24 \mathrm{~h}$. La mayoría (alrededor del $80 \%$ ) de los neonatos fallecidos habían nacido en los hospitales donde se hizo el reporte, sin embargo, también hubo neonatos fallecidos referidos por otros establecimientos de salud de menor complejidad $(15,0 \%)$ y otros nacidos en domicilio $(4,6 \%)$.

La causa de fallecimiento más frecuente en los neonatos fue infección (sepsis neonatal en la mayoría de casos) tanto en Huánuco (34,3\%) como en Ucayali $(29,7 \%)$; más de la mitad de dichas infecciones, en ambas regiones, correspondieron a neonatos prematuros. En Huánuco, también fueron causas básicas de fallecimiento: la prematuridad $(17,9 \%)$, la insuficiencia respiratoria $(17,9 \%)$, la malformación congénita $(16,4 \%)$ y la asfixia (11,9\%). En Ucayali: la malformación congénita $(25,4 \%)$, la prematuridad $(19,5 \%)$, la asfixia $(12,7 \%)$ y la insuficiencia respiratoria $(11,9 \%)$.

Las malformaciones congénitas en los neonatos fallecidos en Huánuco fueron: malformaciones congénitas múltiples (6); defectos del tubo neural (2); gastrosquisis (1); cardiopatía congénita (1) y osteogénesis imperfecta (1). En Ucayali, las malformaciones congénitas identificadas fueron: defectos del tubo neural (10); malformaciones congénitas múltiples (6); cardiopatía congénita (5); atresia del tubo digestivo (3); gastrosquisis (3); síndrome de Edward (1); hernia diafragmática (1), y agenesia de coanas (1).

No hubo asociación significativa entre el peso de nacimiento y el momento del fallecimiento, tampoco entre la edad gestacional al nacimiento y el momento de la defunción en Huánuco (Tabla 5). Sin embargo, fue claro encontrar mayor frecuencia de mortalidad por prematuridad $(31,6 \%)$, malformación congénita $(26,3 \%)$ y asfixia $(21,1 \%)$ en las primeras $24 \mathrm{~h}$ de vida; así como infección $(32,5 \%)$ e insuficiencia respiratoria $(25,0 \%)$ entre el primer y séptimo día de vida. Además, todas las muertes neonatales después del sétimo día fueron por infección.

En Ucayali, por el contrario, sí hubo una asociación significativa entre el peso de nacimiento y el momento del fallecimiento, y entre la edad gestacional al nacimiento y el momento de la muerte $(p<0,05)$ (Tabla 6). Más de
Tabla 4. Características clínicas de los neonatos fallecidos en los hospitales de Huánuco y Ucayali, Perú 2011

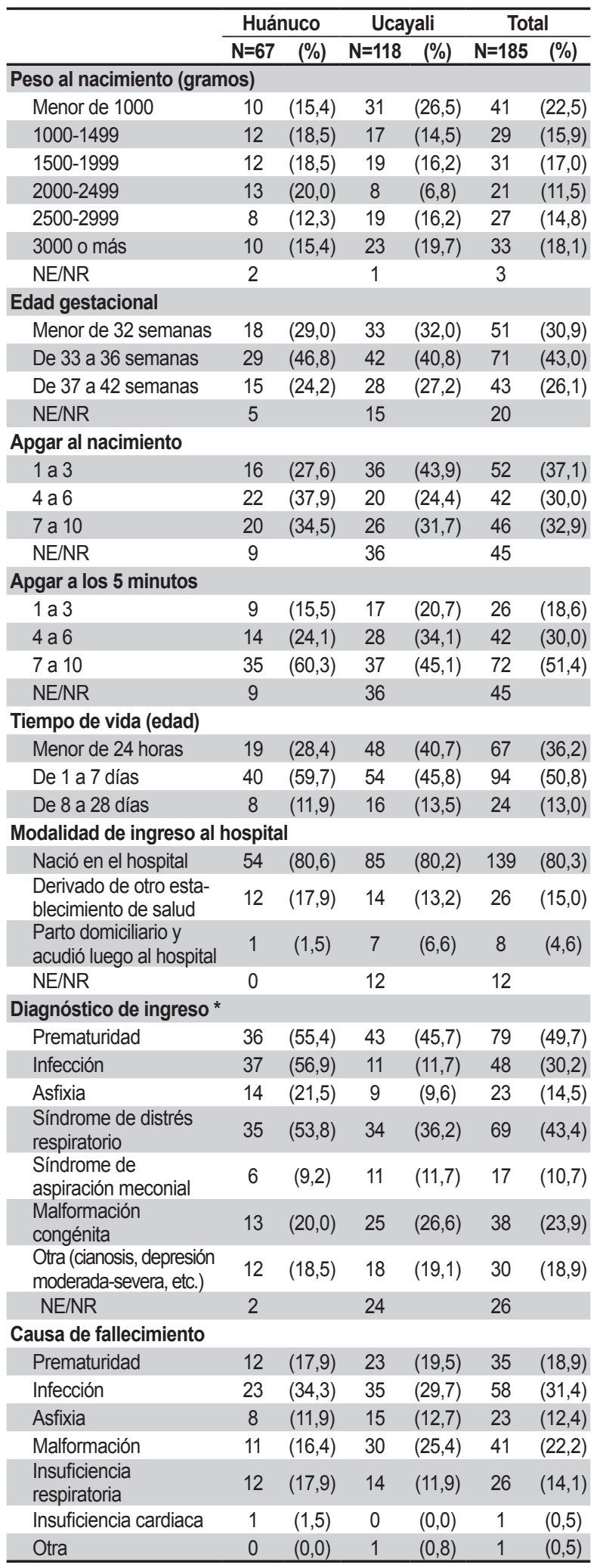

$\mathrm{NE} / \mathrm{NR}=$ no especificado, no registrado.

* Es posible que un mismo neonato haya tenido uno o más diagnósticos de ingreso. 
Tabla 5. Características y causas de fallecimiento en los neonatos y su relación con el momento de fallecimiento en Huánuco, Perú 2011

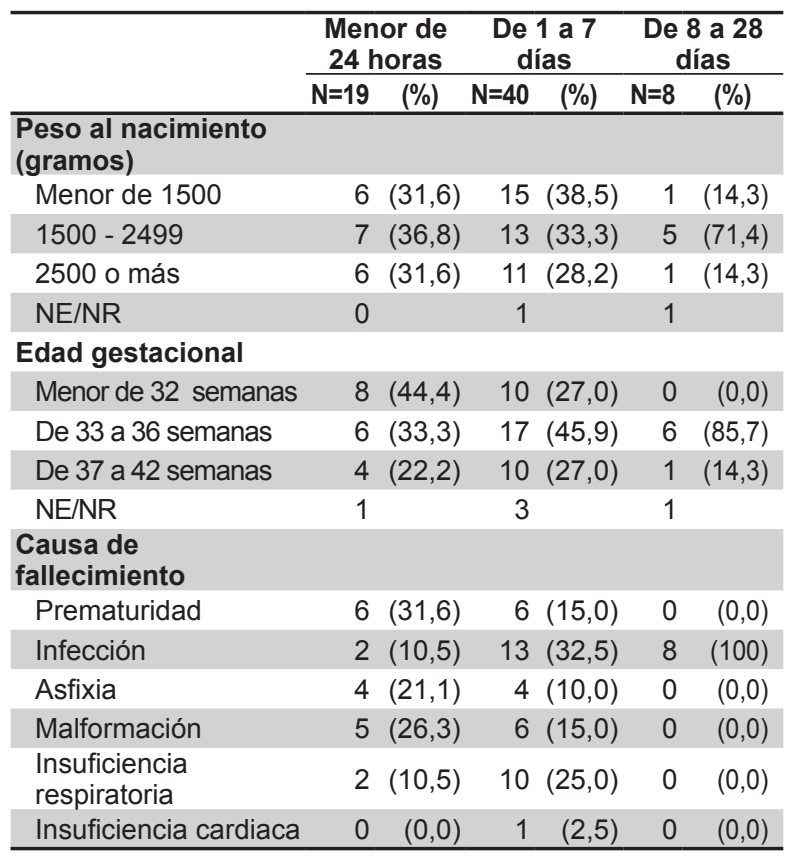

la mitad de los niños fallecidos en las primeras $24 \mathrm{~h}$ tuvieron un peso menor de $1500 \mathrm{~g}$; también, más de la mitad de los niños fallecidos en la etapa neonatal tardía (después del séptimo día) tuvieron peso por encima de los $2500 \mathrm{~g}$. De forma similar, más de la mitad de los niños que fallecieron dentro del primer día de nacidos tenían menos de 32 semanas de edad gestacional. La mortalidad por prematuridad $(39,6 \%)$ y malformación congénita $(35,4 \%)$ predominó dentro de las primeras $24 \mathrm{~h}$ de vida, mientras que la infección se volvió mucho más importante entre el primer y séptimo día $(40,7 \%)$ y después del séptimo día (75\%).

\section{DISCUSIÓN}

La revisión de los registros de defunciones neonatales en el año 2011 en las regiones de Huánuco y Ucayali permitió la identificación de discrepancias en la información sobre la mortalidad neonatal en diferentes niveles, la estimación de tasas de mortalidad neonatal en ambas regiones luego de identificar las fuentes de información más confiables, y la descripción de las principales características de las muertes neonatales registradas en las historias clínicas de los hospitales de referencia de las regiones.

Además del subregistro de muertes neonatales identificado en los registros civiles de las municipalidades provinciales de las regiones Huánuco y Ucayali (problema reportado también en Lima); el estudio encontró discrepancias entre las diferentes áreas involucradas en la vigilancia de la mortalidad neonatal al interior de las DIRESA ${ }^{(9)}$. La falta de estandarización de procedimientos de colección y consolidación de datos de las muertes neonatales explicarían dichas discrepancias. Con el propósito de superar esta limitación, se viene implementando progresivamente el Subsistema de Vigilancia Perinatal y Neonatal, que busca que la información generada por la vigilancia sea de calidad para su uso en el

Tabla 6. Características y causas de fallecimiento en los neonatos y su relación con el momento de fallecimiento en Ucayali, Perú 2011

\begin{tabular}{|c|c|c|c|c|c|c|c|}
\hline & \multicolumn{2}{|c|}{$\begin{array}{c}\text { Menor de } 24 \\
\text { horas }\end{array}$} & \multicolumn{2}{|c|}{ De 1 a 7 días } & \multicolumn{2}{|c|}{ De 8 a 28 días } & \multirow{2}{*}{$p$} \\
\hline & $\mathrm{N}=48$ & (\%) & $N=54$ & $(\%)$ & $\mathrm{N}=16$ & $(\%)$ & \\
\hline \multicolumn{8}{|c|}{ Peso al nacimiento (gramos) } \\
\hline Menor de 1500 & 27 & $(56,3)$ & 18 & $(34,0)$ & 3 & $(18,8)$ & 0,400 \\
\hline $1500-2499$ & 10 & $(20,8)$ & 13 & $(24,5)$ & 4 & $(25,0)$ & \\
\hline 2500 o más & 11 & $(22,9)$ & 22 & $(41,5)$ & 9 & $(56,3)$ & \\
\hline NE/NR & & & 1 & & & & \\
\hline \multicolumn{8}{|l|}{ Edad gestacional } \\
\hline Menor de 32 semanas & 24 & $(52,2)$ & 8 & $(17,8)$ & 1 & $(8,3)$ & 0,002 \\
\hline De 33 a 36 semanas & 15 & $(32,6)$ & 20 & $(44,4)$ & 7 & $(58,3)$ & \\
\hline De 37 a 42 semanas & 7 & $(15,2)$ & 17 & $(37,8)$ & 4 & $(33,3)$ & \\
\hline NE/NR & 2 & & 9 & & 4 & & \\
\hline \multicolumn{8}{|l|}{ Causa de fallecimiento } \\
\hline Prematuridad & 19 & $(39,6)$ & 4 & $(7,4)$ & 0 & $(0,0)$ & $<0,001$ \\
\hline Infección & 1 & $(2,1)$ & 22 & $(40,7)$ & 12 & $(75,0)$ & \\
\hline Asfixia & 6 & $(12,5)$ & 8 & $(14,8)$ & 1 & $(6,3)$ & \\
\hline Malformación & 17 & $(35,4)$ & 10 & $(18,5)$ & 3 & $(18,8)$ & \\
\hline Insuficiencia respiratoria & 5 & $(10,4)$ & 9 & $(16,7)$ & 0 & $(0,0)$ & \\
\hline Otra & 0 & $(0,0)$ & 1 & $(1,9)$ & 0 & $(0,0)$ & \\
\hline
\end{tabular}


diseño y ejecución de intervenciones sanitarias dirigidas al mejoramiento de la salud materna, perinatal y neonatal ${ }^{(10)}$. Previo al cálculo de las tasas de mortalidad neonatal fue necesario identificar la fuente de información más confiable en cada región, es decir, aquella del área de la DIRESA que tuvo menos discrepancia con los registros hospitalarios de muertes neonatales. Las tasas de mortalidad neonatal en Huánuco (14,5 por 1000 nacidos vivos) y Ucayali (12,1 por 1000 nacidos vivos) calculadas para el 2011 están por encima de la tasa estimada a nivel nacional para el mismo año a partir de los datos de la encuesta demográfica y de salud familiar-ENDES 2011 (8 por 1000 nacidos vivos) ${ }^{(3)}$. Las tasas de mortalidad de ambas regiones también superan la mediana mundial publicada por la Organización Mundial de la Salud en el 2011 ( 11 por 1000 nacidos vivos), siendo mayores al promedio de Latinoamérica (9 por 1000 nacidos vivos), pero mucho menores que los estimados en África (34 por 1000 nacidos vivos) y Asia (29 por 1000 nacidos vivos) ${ }^{(1)}$.

Se ha reportado que hay menor riesgo de morbilidad y mortalidad neonatal cuando la madre sigue apropiadamente el control médico en el embarazo, y cuando el parto es atendido por un profesional competente (11). Cada control prenatal representa una oportunidad para identificar complicaciones del embarazo (infecciones, diabetes, preclampsia, etc.) y establecer medidas preventivas e intervenciones oportunas, incrementando las chances de culminar una gestación con un parto seguro ${ }^{(12)}$. Por ello, la asistencia precoz y periódica a los controles prenatales, y el parto institucional son consideradas prácticas clave de cuidado maternoinfantil, y el Ministerio de Salud persigue su universalidad para todas las gestantes del territorio nacional (13). Lamentablemente, aún hay una importante proporción de gestantes que no acuden al control prenatal, con el riesgo que ello conlleva para la madre y el niño. La revisión de historias clínicas hospitalarias mostró que alrededor del $10 \%$ de madres no acuden a control prenatal durante la gestación y, en general, en ambas regiones, el número de controles prenatales que tuvieron las madres no fue óptimo. Por otro lado, si bien la frecuencia de parto institucional fue elevada, la metodología del estudio no permite cuantificar la real proporción de neonatos fallecidos que nacieron en el domicilio y nunca fueron atendidos, ni registrados en un servicio de salud.

Más del $85 \%$ de las muertes en los hospitales de ambas regiones se registraron en el periodo neonatal temprano (primeros 7 días a partir del nacimiento), con una mayor proporción de muertes dentro de las primeras $24 \mathrm{~h}$ en Ucayali $(40,7 \%)$ al comparar con aquellas registradas en Huánuco $(28,4 \%)$. Los porcentajes ligeramente superiores a los señalados por la Organización Mundial de la Salud $(75 \%$ de fallecimientos en la primera semana de vida, con 25 al $50 \%$ de ellos en las primeras $24 \mathrm{~h}$ ), estarían relacionados con la alta frecuencia de algunas condiciones en los neonatos que incrementan la mortalidad neonatal temprana como: la prematuridad y el bajo peso al nacer, entre otras ${ }^{(14)}$.

Tanto en Huánuco como en Ucayali se encontró un alto porcentaje de neonatos con bajo peso al nacer dentro de los fallecidos, y la gran mayoría de ellos también fueron prematuros. El bajo peso al nacer es considerado uno de los indicadores más importantes para determinar las posibilidades del recién nacido de sobrevivir y tener un crecimiento sano. Según las estimaciones realizadas por UNICEF, aproximadamente $14 \%$ de todos los recién nacidos nacen con un peso inferior al normal. Los neonatos con bajo peso y especialmente aquellos con peso inferior a $1500 \mathrm{~g}$ (muy bajo peso al nacer) determinan hasta el $60 \%$ de la mortalidad neonatal ${ }^{(15)}$. Los que sobreviven tienen disminuidas las funciones del sistema inmune y corren mayor riesgo de padecer, posteriormente, algunas enfermedades como diabetes y diversas cardiopatías. Tienen también propensión a seguir malnutridos y a tener menores coeficientes de inteligencia, además de discapacidades cognoscitivas. El peso al nacer refleja la experiencia intrauterina, siendo también un buen indicador del estado de salud y la nutrición de la madre ${ }^{(4,16)}$. Desafortunadamente, el registro variable en las historias clínicas (con datos incompletos en varias de ellas) en relación a la presencia de enfermedades crónicas concomitantes en la madre (diabetes, hipertensión arterial, enfermedades infecciosas crónicas, anemia, etc.), hábitos nocivos, estado nutricional y complicaciones del embarazo (eclampsia, patologías placentarias), no permitió establecer asociaciones entre estas variables y el bajo peso al nacer en los neonatos fallecidos.

La proporción elevada de neonatos con edad gestacional menor de 32 semanas, prematuridad extrema, y con muy bajo peso al nacer genera interrogantes sobre si las regiones estudiadas tienen la capacidad apropiada para el manejo de este grupo de pacientes complejos. Se requiere una evaluación de la capacidad resolutiva de los hospitales para determinar si cuentan con servicios ginecoobstétricos y neonatales apropiados, así como con unidades de cuidados intermedios/ intensivos neonatales con equipos médicos básicos, insumos médicos, medicamentos, exámenes auxiliares y de imágenes, protocolos de manejo actualizados, y recursos humanos competentes y con experiencia.

La revisión de las causas de muerte en los registros consolidados de las DIRESA y, posteriormente, en las historias clínicas hospitalarias evidenció una falta de estandarización en la determinación de las causas de mortalidad neonatal, empleándose inapropiadamente y en 
repetidas oportunidades algunos términos de entidades nosológicas genéricas de la décima Clasificación Internacional de Enfermedad (CIE-10) (ejemplo: trastornos respiratorios y/o vasculares) que esconden las causas reales de muerte en un recién nacido. Por ello, las causas de muerte neonatal en el presente estudio, identificadas luego de una minuciosa revisión de historias clínicas, difieren de los datos oficiales del Ministerio.

Si bien en ambas regiones predominó la infección (sepsis neonatal) como principal causa básica de mortalidad neonatal, esta se dio mayoritariamente en neonatos con prematuridad y bajo peso al nacer. La inmadurez de las defensas del neonato prematuro, sobre todo si tiene bajo peso al nacer, se constituye en un factor predisponente para el desarrollo de la infección (17). Es importante mencionar que las infecciones identificadas en el estudio como causa de muerte en el periodo neonatal temprano, señalan una alta probabilidad de transmisión por contacto del neonato con gérmenes en el canal vaginal, originando, la mayoría de veces, un tipo de sepsis de curso clínico grave, fulminante y multisistémico ${ }^{(18)}$.

Por ser los hospitales seleccionados centros de referencia de las regiones, es mucho más frecuente en ellos la atención de embarazos de riesgo identificados en los mismos hospitales o referidos desde otros establecimientos de salud. Esto explicaría por qué las tasas de muertes neonatales en los hospitales de forma individual son mayores a las estimadas para cada región a la que pertenecen, y por qué se encontró una elevada frecuencia de neonatos fallecidos con malformaciones congénitas. Sin embargo, llama la atención especialmente en Ucayali que la mayoría de malformaciones fueron defectos del tubo neural, enfermedad asociada a deficiencia de folatos durante el embarazo (19,20). Si bien no se encontró en las historias clínicas revisadas información de consumo de ácido fólico durante el embarazo, reportes recientes en áreas con pobreza extrema del Perú señalan que la administración de ácido fólico en los controles prenatales es poco frecuente ${ }^{(21)}$.

Mantener la actual tendencia decreciente en la mortalidad infantil y aproximarse más al cumplimiento de las metas del Milenio, requiere un abordaje estratégico en el periodo de mayor vulnerabilidad del niño que es el periodo neonatal (2). Información oportuna y confiable sobre la morbilidad y mortalidad neonatal y sus determinantes, obtenida de un sistema de vigilancia bien estructurado, complementada con investigaciones operativas y científicas que ayuden a comprender mejor los resultados de la vigilancia o superar las limitaciones de esta, debe permitir el diseño de intervenciones integrales en el continuo: embarazo-parto-periodo posnatal. El presente estudio descriptivo evidenció tasas de mortalidad neonatal superiores a la media nacional para el año 2011 en las regiones seleccionadas, tasas que fueron estimadas luego de un trabajo laborioso para identificar la mejor fuente de información sobre muertes neonatales en cada región. La mayoría de neonatos atendidos en los hospitales de referencia de las regiones fallecieron en los primeros 7 días de vida, fueron prematuros o tuvieron bajo peso al nacimiento; identificándose como causas básicas principales para las muertes: infección, prematuridad y malformación congénita.

Fuentes de financiamiento: la investigación contó con el apoyo del Programa de Apoyo a la Reforma del Sector Salud del Ministerio de Salud, proyecto de inversión pública financiado con recursos del tesoro público y endeudamiento externo proveniente del Banco Interamericano de Desarrollo (BID) y el Banco Mundial (BM).

Contribuciones de autoría: JEVH, LKF, TGPQ, RHLR, WEVV participaron en la concepción y el diseño del estudio, además de la recolección de datos. Todos participaron en el análisis e interpretación de los datos. JEVH y AMRA redactaron el artículo y todos los autores revisaron los borradores y la versión final, realizando importantes contribuciones.

Conflictos de interés: en la presente investigación no existe conflictos de interés.

\section{REFERENCIAS BIBLIOGRÁFICAS}

1. Organización Mundial de la Salud. Estadísticas Sanitarias Mundiales 2012. Ginebra: OMS; 2012.

2. Instituto Nacional de Estadística e Informática. Perú: Indicadores de Resultados de los Programas Estratégicos, 2000 - 2012 [Internet]. Lima: INEI; 2013 [20 de agosto de 2013]. Disponible en: http://www.midis.gob.pe/dgsye/ datal/files/enic/eje1/documentotecnico/ENDES-PPR2012.pdf

3. Instituto Nacional de Estadística e Informática. Encuesta Demográfica y de Salud Familiar 2012 [Internet]. Lima: INEI; 2013 [20 de agosto de 2013]. Disponible en: http:// proyectos.inei.gob.pe/endes/2012/

4. World Health Organization; UNICEF. Countdown to 2015 decade report (2000-2010) with country profiles: taking stock of maternal, newborn and child survival. Ginebra: WHO; 2010

5. Lawn JE, Cousens S, Zupan J; Lancet Neonatal Survival Steering Team. 4 million neonatal deaths: when? Where? Why? Lancet. 2005 Mar 5-11;365(9462):891-900.
6. UNICEF, Agencia Española de Cooperación Internacional para el Desarrollo. Estudio sobre dimensión cuantitativa y concepciones y cuidados comunitarios de la salud del recién nacido, en un área rural andina $\mathrm{y}$ amazónica del Perú. Capítulo I: Componente Estadístico. Lima: UNICEF; 2010.

7. Perú, Ministerio de Salud del Perú. Estudio de Factibilidad del Programa de Apoyo a la Reforma del Sector Salud - PARSALUD II [Internet]. Lima: 
PARSALUD; 2008 [citado el 20 de agosto de 2013].

8. Organización Panamericana de la Salud; Organización Mundial de la Salud. Clasificación Estadística Internacional de Enfermedades y Problemas Relacionados con la Salud, Décima Revisión (CIE-10), Vol. 2 "Manual de instrucciones". 3ra ed. Washington, DC: OPS; 2003.

9. Espíritu N, Sacieta L, Pantoja L. Discrepancias en el registro de la mortalidad perinatal en Lima y Callao según fuente de información. Rev Peru Med Exp Salud Publica. 2007;24(4):363-9.

10. Perú, Ministerio de Salud. Subsistema nacional de vigilancia epidemiológica perinatal y neonatal. Lima: MINSA; 2009.

11. Harvey SA, Blandón YC, McCawBinns A, Sandino I, Urbina L, Rodríguez C, et al. Are skilled birth attendants really skilled? A measurement method, some disturbing results and a potential way forward. Bull World Health Organ. 2007 Oct;85(10):783-90.

12. Say L, Raine R. A systematic review of inequalities in the use of maternal health care in developing countries: examining the scale of the problem and the importance of context. Bull World Health Organ. 2007 Oct;85(10):812-9.
13. Perú, Ministerio de Salud. Documento Técnico: Promoción de prácticas y entornos saludables para el cuidado infantil. Lima: MINSA; 2011.

14. Organización Mundial de la Salud. Reducción de la mortalidad en la niñez [Internet]. Ginebra: OMS; 2012 [citado el 20 de agosto de 2013]. Disponible en: http://www.who.int/ mediacentre/factsheets/fs178/es/

15. UNICEF. Estado Mundial de La Infancia: Conmemoración de los 20 años de la convención sobre los derechos del Niño [Internet]. Nueva York: UNICEF: 2010 [citado el 20 de agosto de 2013]. Disponible en: http://www.unicef.org/ spanish/rightsite/sowc/fullreport.php

16. Black RE, Allen LH, Bhutta ZA, Caulfield LE, de Onis M, Ezzati M, et al. Maternal and child undernutrition: global and regional exposures and health consequences. Lancet. 2008 Jan 19;371(9608):243-60. doi: 10.1016/ S0140-6736(07)61690-0.

17. Barros FC, Bhutta ZA, Batra M, Hansen TN, Victora CG, Rubens CE, et al. Global report on preterm birth and stillbirth (3 of 7): evidence for effectiveness of interventions. BMC Pregnancy Childbirth. 2010 Feb 23;10 Suppl 1:S3. doi: 10.1186/1471-239310-S1-S3.

18. López Saster JB, Coto Cotallo GD, Fernández Colomer B; Grupo de Hospitales Castrillo. Neonatal sepsis of vertical transmission: An epidemiological study from the "Grupo de Hospitales Castrillo". J Perinat Med. 2000;28(4):309-15.

19. Mahomed K. Folate supplementation in pregnancy. Cochrane Database Syst Rev. 2000;(2):CD000183.

20. Czeizel AE, Dudás I, Vereczkey A, Bánhidy F. Folate deficiency and folic acid supplementation: the prevention of neural-tube defects and congenital heart defects. Nutrients 2013 Nov 21;5(11):4760-75. doi: 10.3390/ nu5114760.

21. Velásquez J, Solis L, Vigo W, Rosas A, Giusti P, Alfaro P, Cabrera H. Evaluación de las prácticas de cuidado materno-infantil en áreas con pobreza extrema del Perú. Enviado a Rev Peru Med Exp Salud Publica. 2014;31(2): 243-53.

Correspondencia: José Velásquez Hurtado Dirección: Av. Paso de los Andes 681. Pueblo Libre, Lima 21 - Perú.

Correo electrónico: jose.velasquez@upch.pe

\title{
Consulte la versión electrónica de la Revista Peruana de Medicina Experimental y Salud Pública en
}

\author{
wWw.scopus.com
}

TM
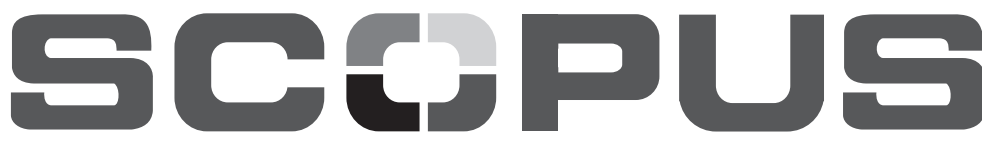